makes politics and economics not just controversial, but interesting.

Inequality is one of the biggest items on the agendas of both of these disciplines. Few people are likely to speak in favour of inequality as such, but in stereotypical terms the political right defends wealth as a reward for hard work, whereas the left deplores a society in which, as economist Joseph Stiglitz has said of the United States, "1 percent of the people take nearly a quarter of the nation's income". It seems an unavoidable truth that a free-market capitalist system will create wealth inequality; to a free-market fundamentalist who sees markets as meritocratic optimizers of efficiency and resource utilization, that is not only necessary but moral. Under that philosophy, by intervening in the market in the hope of making the outcome 'fairer', we only throw a spanner in the works.

Yet even if one accepts some inequality as a necessary evil, there are options beyond laissez-faire. How, and how strenuously, governments and legislators should attempt to limit the extent of wealth inequality crudely measured by the Gini coefficient, which quantifies the statistical dispersion of income distribution - is currently a hotly disputed matter. Should companies and banks be restricted in what they can pay their chief executives? Should taxes aim to inhibit or reduce the perpetuation of inherited wealth? Or is all this crypto-communist social engineering?

The strongest argument for such measures is not that it makes things more 'fair' (although meritocratic defences of free-market inequalities should surely at least demand a level playing field). Rather, it is that gross wealth inequality is socially corrosive. It polarizes attitudes, foments unrest (see, for example, the Occupy movement) and degrades trust and cooperation. At face value, a study published online this week in Nature supports that view — but with an added twist.

In the study, groups of volunteers played a simple economic game involving cooperation (a "public goods game"), in which they could lose or gain wealth through voluntary redistribution within social networks that started with three different levels of inequality (A. Nishi et al. Nature http://dx.doi.org/10.1038/nature15392;2015). Crucially, in some games the wealth of participants was made visible to others, whereas in others it was kept hidden. For "invisible" wealth conditions, the games tended to converge on a fairly low Gini coefficient,

but "visible" wealth produced higher (and less stable) average Gini coefficients. This result was exacerbated when the initial inequality was greater. In other words, simply hiding wealth decreased the wealth disparity in otherwise identical games and networks.

Still more importantly, visible wealth reduced the overall cooperation and interconnectedness of the social network, and in fact led to lower total wealth. As the authors say: "it is not inequality per se that

"Inequality is not solely down to market mechanisms, but also responds in subtle ways to our own dispositions." is so problematic, but rather visibility" of that inequality. This fits with the established idea that it is relative, not absolute, differences in wealth that compromise happiness and promote discord: we resent what our neighbours have and we don't. What grates is not knowing that others have more than us, but seeing that difference ostentatiously displayed.

It is dangerous, however, to think that these laboratory experiments can be extrapolated into a political or moral message for the real world. They invite us to frown on bling and the champagne-drenched excesses of financiers, but we should be cautious about their implications, even (or especially) if they flatter our preconceptions. Besides, there is scope here for upsetting both ends of the political spectrum. Right-wingers might deplore an injunction to hide one's wealth, compromising personal freedom - isn't it up to us how we spend our money? Left-wingers might dislike the idea of being relaxed about inequality as long as it is kept out of sight - and, anyway, might that not provoke a climate of secrecy and suspicion?

For now, the results should simply inform and broaden the discussion. They show, for example, that inequality is not solely down to market mechanisms, but also responds in subtle ways to our own dispositions. Above all, the findings are a reminder, along with related behavioural experiments on the role of punishment in public-goods games, that John Maynard Keynes's "animal spirits" are an irreducible part of what shapes a market economy. It is time to lay the idea of the rational Homo economicus to rest.

\section{Loaded language}

There can be more to a question than appears at first sight.

W illiam Burroughs, the infamous US writer and author of Naked Lunch, had a typically counter-culture approach to seeking knowledge: "Your mind will answer most questions if you learn to relax and wait for the answer."

If only it were that easy for the rest of us. Instead, to ask a question is harder than it might seem. British Prime Minister David Cameron discovered this last month when the UK Electoral Commission told him to change the wording of a proposed question for the country's referendum on membership of the European Union.

Cameron's suggestion - "Should the United Kingdom remain a member of the European Union?" - was a classic example of what linguists call acquiescence bias. Take the Burroughs route and relax, and the answer to such a question that comes to mind more often than not is to stick with the status quo. Rejecting something is more difficult.

If that was Cameron's intention, then his plan has been rumbled. The question will now have the extra clause at the end: "or leave the European Union?" To answer that one, citizens must now make more of a cognitive effort, and that should remove the chance for bias.

Cameron's linguistic nudging was more subtle than most attempts to bias questions. Lawyers and politicians tend to be fans of more explicit tricks of language. There is the classic loaded question - when did you stop beating your wife? - which presupposes guilt; and the pernicious influence of the hypothetical question. During the 2000 US election campaigns, South Carolina voters were asked: would you be more likely or less likely to vote for John McCain for president if you knew he had fathered an illegitimate black child??"

Researchers have found that the way a question is phrased can alter how people remember incidents. Witnesses asked how quickly cars were travelling when they "smashed" are more likely to imagine that they saw broken glass on the ground than others told that the vehicles simply "bumped" into each other or "collided". They were also more likely to say that the cars were travelling at higher speed.

Scientists have a particular relationship to questions. Turned into testable null hypotheses, questions are at the heart of the scientific method. Allied with proper experimental design and robust statistical analysis, they can be answered with confidence - or not.

Some answers are known before the question is asked; other questions are genuine calls for information. Some want to benefit the questioner and others to empower those who answer it. How to judge? In all areas - politics and science included - the best questions are simple and to the point. So who knows what the residents of Quebec thought when confronted with the following for their referendum on independence in 1995:

"Do you agree that Quebec should become sovereign, after having made a formal offer to Canada for a new economic and political $\rightarrow$ NATURE.COM To comment online, click on Editorials at: go.nature.com/xhunqv partnership, within the scope of the bill respecting the future of Quebec and of the agreement signed on June 12, 1995?"

The 'No' vote won with 50.6\%. 'Don't know's were not recorded.. 